\title{
Locacorten Vioform Ototoxicity Upon Guinea Pig Middle Ear Application
}

\author{
Owen Woods and Issam Saliba \\ Division of Otolaryngology-Head and Neck Surgery, Sainte-Justine University Hospital Center, University of Montreal, Montreal, Canada
}

Received July 28, 2017

Revised November 20, 2017

Accepted December 5, 2017

\section{Address for correspondence Issam Saliba, MD, FRCSC \\ Division of Otolaryngology- Head and Neck Surgery, \\ Sainte-Justine University \\ Hospital Center, \\ University of Montreal, \\ 3175, Côte Sainte-Catherine, \\ Montréal (QC) H3T 1C5, Canada \\ Tel +514-345-4857 \\ Fax +514-507-9014 \\ E-mailissam.saliba@umontreal.ca}

Background and Objectives: Locacorten Vioform (Novartis UK) is frequently prescribed for otomycosis. Its component, Clioquinol, also has anti-bacterial properties. Up to this point, its ototoxic potential has not been evaluated. Our objective aims to evaluate Locacorten Vioform's potential ototoxicity when applied directly to the middle ear cavity. Materials and Methods: We performed an experimental prospective animal study in our animal research center with 20 Hartley guinea pigs divided into 2 groups. The first group (experimental) was treated with Locacorten Vioform in one ear and with a physiologic saline solution in the other. The second group (positive control) was treated with concentrated gentamycin in one ear and physiologic saline in the other. Auditory brainstem response measurements were obtained before and after three sets of injections. Statistics were analyzed using a variance analysis with repeated measures. The histological state of cochlear outer hair cells was compared between the two groups using scanning electron microscopy. Results: Average hearing loss in ears treated with Locacorten Vioform was $32.1 \mathrm{~dB}$, compared with a $2.5 \mathrm{~dB}$ average loss in the saline-treated ears. Ears treated with gentamycin lost an average of $33.0 \mathrm{~dB}$. There were clinically and statistically significant differences between the two ears of the guinea pigs in both groups $(p<0.001)$. Scanning electron microscopy revealed severe pericochlear and cochlear inflammation and ossification in the Locacorten Vioform-treated ears. Gentamycin caused significant destruction of outer hair cell architecture. Conclusions: Locacorten Vioform induces a hearing loss similar to that caused by gentamycin when applied directly to the middle ear of a guinea pig model. Electron microscopy indicates a pericochlear and cochlear inflammatory reaction with ossification.

J Audiol Otol 2018;22(2):75-79

\section{Introduction}

Otomycosis is a common cause of otalgia and aural fullness, particularly in patients recently treated with a topic antibacterial agent. It is defined as a fungal infection of the external or middle ear, or of an open mastoid cavity. Patients with chronic otitis media are at increased risk of developing otomycosis. The use of topical quinolones has also been shown to increase its prevalence [1].

As with all fungal infections, a hot and humid environment

This is an Open Access article distributed under the terms of the Creative Commons Attribution Non-Commercial License (http://creativecommons.org/licenses/by-nc/4.0/) which permits unrestricted non-commercial use, distribution, and reproduction in any medium, provided the original work is properly cited. predisposes to otomycosis. Most patients present with unilateral ear pain and aural fullness, with possible persistent discharge and mild hearing loss. Physical examination varies depending on the offending agent and extent of disease, but typically reveals a white crust lining the external auditory canal with a normal tympanic membrane (TM). Culture reveals' Candida albicans, Candida parapsilosis, and Aspergillus fumigatus in $95 \%$ of cases [2]. When black crusts are identified, Aspergillus infection should be suspected. Mainstay of treatment consists of cleaning the infected ear, keeping it dry and applying topical antifungal cream or ointment.

Not all antifungal agents are appropriate for otomycosis treatment. Acetic acid and gentian violet have known ototoxic potential and should therefore be avoided $[3,4]$. Several alter- 
native agents have proven their safety in animal studies, including ciclopirox, clotrimazole, miconazole, and nystatin [4-7].

Locacorten Vioform (Novartis UK, London, UK) is a topical antifungal agent with three components: clioquinol, flumethasone and a polyethylene glycol vehicle. Its antibiotic component, clioquinol, inhibits bacterial and fungal DNA structure and function [8]. Though studies report the use of Locacorten Vioform going as far back as 1966, no English-language study has reported its efficacy or safety [9].

The goal of this study is to evaluate Locacorten Vioform's ototoxic potential when exposed to the middle ear of a guinea pig model.

\section{Materials and Methods}

\section{Animals}

Twenty Hartley guinea pigs were included in our study. We prefer this species because of their accessible cochlea, their functional anatomy which resembles that of humans, their widespread use in similar studies and finally because of our experience with this species in our laboratory.

Animals were cared for in accordance with the guidelines of the Canadian Council of Animal Care. The study was approved by our institutional review board and follows the standards of the institutional animal care committee (c-461).

\section{General procedures and group assignment}

The experiment was performed over eight weeks, beginning with group assignation and ending with scanning electron microscope (SEM) interpretation.

Each guinea pig was randomly assigned to one of two groups: an experimental group (group I) and a positive control group (group II). Animals from group I received Locacorten Vioform (flumethasone pivalate $0.02 \%$ and clioquinol $1 \%$ ) injections in one randomly selected ear and physiological saline $(0.9 \% \mathrm{NaCl})$ injections in the other. Animals from group II received gentamycin $(40 \mathrm{mg} / \mathrm{mL})$ injections in one randomly selected ear and physiological saline in the other. Each contralateral ear was thus used as a negative control, allowing us to reduce the number of animals required and minimize the impact of variability between animals.

\section{Anesthesia protocol}

Anesthesia was induced by placing the animals in a plastic container with two entrance ports and one exit port. Oxygen and isoflurane $1 \%$ were administered in parallel systems and were ventilated out through the exit port. When animal was placed in the induction chamber and oxygen flowmeter adjusted to approximately $0.5-1.0 \mathrm{~L} / \mathrm{min}$, isoflurane vaporizer was regulated to approximately $3-5 \%$ for induction and then approximately $1-3 \%$ for maintenance. In order to reduce hypothermia related to anesthesia, we used a thermal support until the animal is fully recovered from anesthesia [10]. All manipulations, including middle ear injections and animal sacrifice were performed under general anesthesia. The guinea pigs had spontaneous breathing but did not react to stimulus. Auditory brainstem response (ABR) recordings required us to maintain anesthesia with a mask outside of the container. Each animal's adequate recovery from anesthesia was assured before leaving it unsupervised.

\section{Middle ear injections}

We began our experiment with a microscopic evaluation of each guinea pig's TM. Debris was cleared as needed. Each animal then passed an ABR test within the first week. ABR protocol details are described below.

We then injected approximately $0.1 \mathrm{cc}$ of the experimental solution into each ear, or enough to fill the middle ear cavity, using a Whitacre spinal needle (BD, Middletown, NY, USA). Each injection as described above was repeated every second day for a total of three sets of injections.

At this point we waited a total of two weeks before proceeding with further tests. This waiting time was considered necessary in order to minimize the amount of residue present at the round window niche, which if considerable could interfere with ABR readings as well as SEM analysis.

Our second ABR recordings were performed during week 5. A thorough cleaning and drying of each ear was performed the day before the recordings. It is important to note that all TM were normal and no perforation was identified.

\section{Auditory brainstem response}

All ABR tests were performed with the same Nicolet Bravo $^{\mathrm{TM}}$ device (Nicolet Bravo System; Nicolet Biomedical, Madison, WI, USA). The same room of the Experimental Surgery Center was used for all tests. Electrodes were inserted subcutaneously. The non-inverting electrodes were placed directly above the left and right mastoid processes, the inverting electrode was placed at the apex and the ground electrode was placed in the middle of the animal's back. Impedance was verified and accepted at values inferior to $3.0 \mathrm{k} \Omega$.

We used tone burst stimuli emitted through TIP-300 (Biologic Corp., Madison, WI, USA) earphones to record differences across a range of frequencies. Tone bursts at 2000 , $4,000,6,000$, and $8,000 \mathrm{~Hz}$ were generated with condensation polarity. Amplitude thresholds were found to the nearest $5 \mathrm{~dB}$. A total of 1,500 sweeps were averaged out with each recording. To be considered a positive reading, a visible wave $\mathrm{V}$ needed to 
be reproducible beyond a doubt on a minimum of two stimulations with the same amplitude. ABR values are provided as absolute dB levels.

\section{Animal sacrifice and preparation}

All animals were sacrificed by guillotine under general anesthesia. For initial dissection we focused on exposing the middle ear by removing the residual TM and the cartilaginous portion of the external auditory canal. Fixation was accomplished by immersing the guinea pig heads in a $2.5 \%$ gluteraldehyde solution for 5 days.

Decalcification was performed using a concentrated hydrochloric acid solution (normal 1) for eight days.

Temporal bone dissection was performed at the end of this process. The bone was considerably softened and easy to section with a standard 15-gauge scalpel. We began by isolating the temporal bone from the rest of the cranium and soft tissue. Careful dissection was required to locate the cochlea and separate it from the temporal bone without damaging it. In order to view rows of ciliated hair cells, transverse cuts through the cochlea were made. One section was obtained per turn of the cochlea, giving us four specimens to analyse from each ear.

\section{Scanning electron microscopy}

These sections of each cochlea were then analyzed with our research center's SEM (JSM-6460LV, JEOL, Tokyo, Japan) at week 7 after the last injection. All sections were evaluated for preservation or destruction of cochlear hair cells.

\section{Statistical analysis}

Before beginning our study, we determined the number of animals necessary to find a clinically significant difference $(10 \mathrm{~dB})$ with sufficient statistical power using the method of Snedecor and Cochran [11].

To interpret ABR results, we performed a variance analysis with repeated measures using a four-way analysis of variance. Group, injection product, time (before and after injections) and frequency were considered independently. Results were computed using SPSS version 20 (IBM Corp., Armonk, NY, USA).

\section{Results}

All TMs were normal at the beginning of the study. One guinea pig was excluded after initial ABR evaluation for profound unilateral hearing loss.

\section{Auditory brainstem response}

\section{Descriptive results}

Initial ABR testing yielded similar results between the two groups. Average threshold values across all tested frequencies ranged between $25.6 \pm 2.7 \mathrm{~dB}$ and $27.4 \pm 10.5 \mathrm{~dB}$. After our three sets of injections in each ear, values greatly differed according to the group.

In group I, Locacorten Vioform-treated ears had a threshold of $58.1 \pm 9.6 \mathrm{~dB}$, compared with $29.9 \pm 11.4 \mathrm{~dB}$ in contralateral normal saline-treated ears. In group II, gentamycintreated ears had a threshold of $58.6 \pm 29.6 \mathrm{~dB}$ compared with $29.3 \pm 6.6 \mathrm{~dB}$ in contralateral normal saline-treated ears. Note the high standard deviation in the gentamycin group, which we will discuss in detail below. Results are illustrated in Fig. 1, 2. In addition, in the Locacorten Vioform and gentamicin groups, all the frequencies are affected and there is non spared frequencies.

\section{Statistical analysis}

Variance analysis revealed no interaction between our four

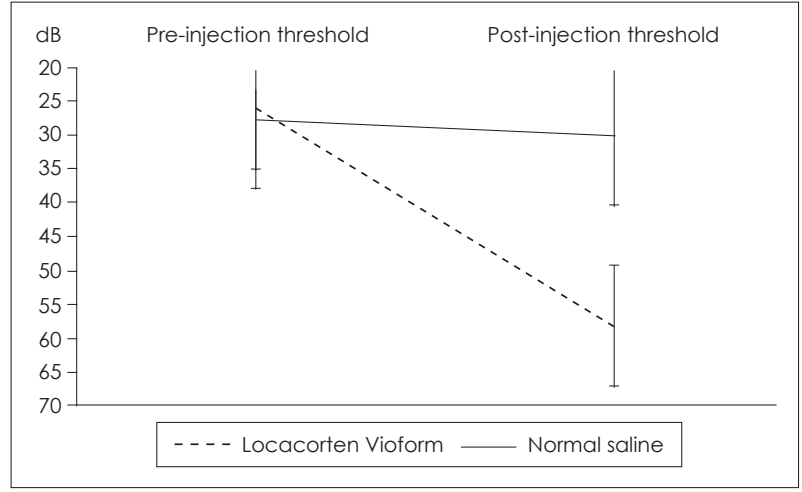

Fig. 1. Auditory brain stem average threshold across all tested frequencies $(2,000,4,000,6,000$, and $8,000 \mathrm{~Hz})$ for the groups Locacorten Vioform and normal saline.

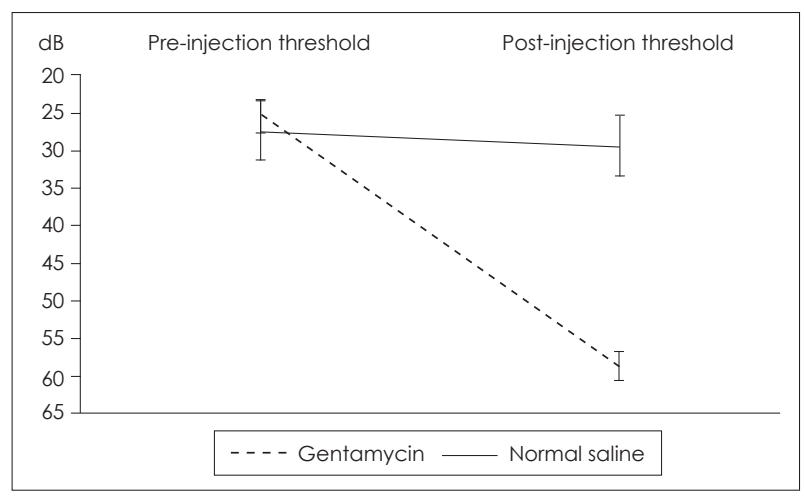

Fig. 2. Auditory brain stem average threshold across all tested frequencies $(2,000,4,000,6,000$, and $8000 \mathrm{~Hz})$ for the groups gentamycin and normal saline. 
measured variables. No statistically significant difference was found between Locacorten Vioform and gentamycin-treated ears $(p=0.782)$. Between these two groups, average hearing loss was $30.3 \mathrm{~dB}$, with a $95 \%$ confidence interval of $18.4 \mathrm{~dB}$ to $42.3 \mathrm{~dB}$. Both of these groups, however, differed significantly from their normal-saline counterparts $(p<0.001)$.

\section{Scanning electron microscopy}

After decalcification, all cochleae were easily prepared with transverse cuts through each turn, with the exception of Locacorten Vioform-exposed cochleae. These were enveloped with a thick layer of inflammatory tissue and ossification, making transverse cuts impossible. An example of such a specimen is shown in Fig. 3.

Normal saline-exposed cochleae showed normal outer hair cell architecture of all three layers at every turn. Gentamycinexposed cochleae, on the other hand, showed marked destruction of these outer hair cells. No significant residue was found in any specimen. Examples of images from each group are shown in Fig. 4.

\section{Discussion}

After proper cleaning and drying of the ear, topical antimicrobial therapy is the most important element in the treatment of otomycosis. Ideally, agents should be safe when exposed to the middle ear, especially in the presence of TM perforation or of a mastoid cavity. Previous studies have been published reviewing the ototoxic potential of several of these agents [7]. While gentian violet and acetic acid are known to have ototoxic properties, clotrimazole, ciclopirox, miconazole and nystatin appear to be safe based on animal studies. With this study, we conclude that Locacorten Vioform is now the

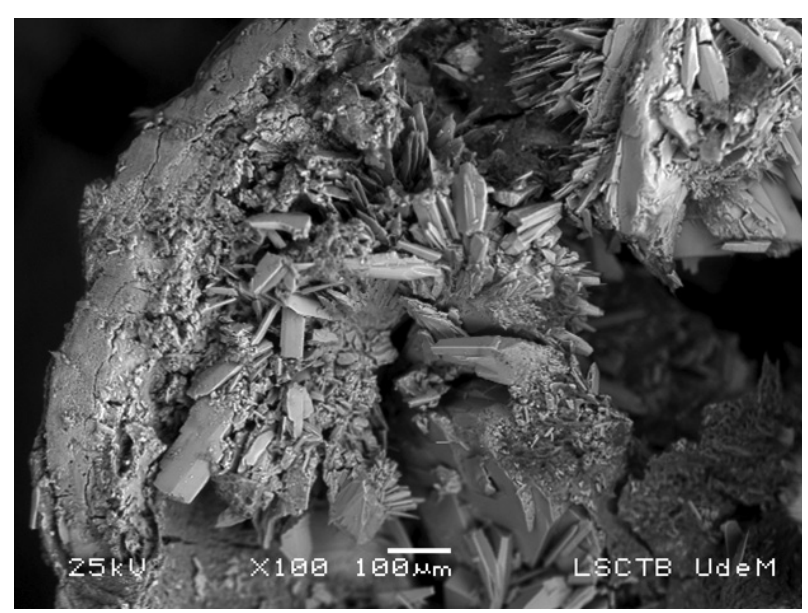

Fig. 3. Guinea pig cochlear ossification following exposure to topical Locacorten Vioform. third known ototoxic antimycotic agent.

Studies evaluating the efficacy of Locacorten Vioform are extremely limited. In fact, the only English-language study, published in 2001 by a Nigerian group, reports only $61 \%$ clinical efficacy [12]. In contrast, in a similar study, Than, et al. reported an $80 \%$ success rate within seven days of initiating Nystatin therapy [13]. Independently of its ototoxicity, evidence for the utility of Locacorten Vioform treatment is lacking.

Another application of Locacorten Vioform has been more recently described. Describing a new technique for tympanoplasty graft preparation, List, et al. [14] recommend immersing a perichondral graft in Locacorten Vioform after harvest. The advantage they report is a stiffening of the graft, which increases the ease of its manipulation for an underlay technique. Though the quantity of Locacorten Vioform exposed to the middle ear in these patients would appear to be minimal, we suggest based on the results of our study to avoid its use in any otologic surgery whenever possible.

Upon analysis of our results, we noted a very high standard deviation in ABR results in ears treated with gentamy-
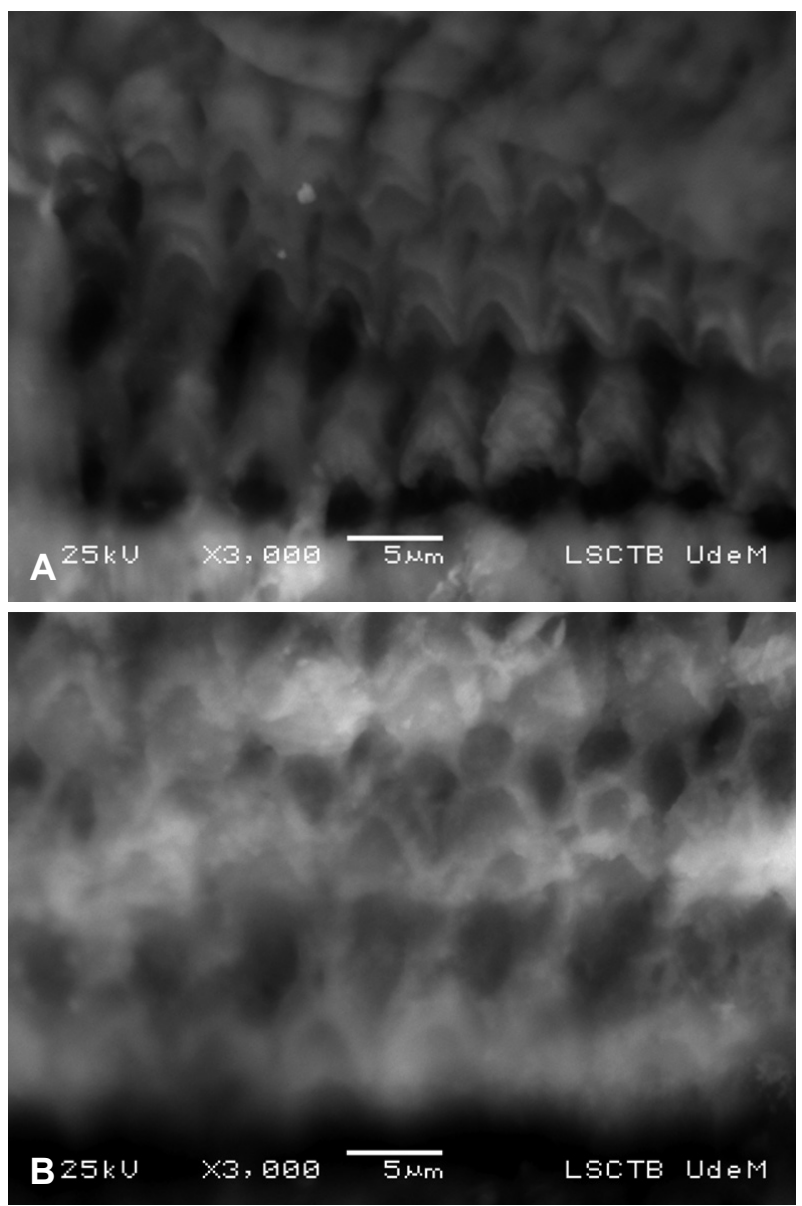

Fig. 4. Scanning electron microscope images of cochlear outer hair cells exposed to normal saline (A) and gentamycin (B). Note the loss of cell architecture in the gentamycin group. 
cin (29.6 dB), compared with Locacorten Vioform-treated ears $(9.6 \mathrm{~dB})$. Further review of our data shows that of ten ears treated with gentamycin, four resulted in profound hearing loss (range $=87.5-97.5 \mathrm{~dB}$ ), while the six others resulted in moderate hearing loss $($ range $=31.3-45.0 \mathrm{~dB}$ ). This bimodal distribution is not surprising, as genetic predisposition to aminoglycoside ototoxicity is well described. A mutation of mitochondrial DNA that codes for the $12 \mathrm{~S}$ ribosomal RNA subunit may be responsible for this phenomenon in humans [15]. To our knowledge, this genetic predisposition has not previously been reported in the guinea pig.

Scanning electron microscopy was very telling in this study, as our images corresponded well with ABR testing. The ossification that we observed in the Locacorten Vioform group is similar to that observed in a cochlea of a patient having suffered from bacterial meningitis. This intense inflammatory reaction is most like due to glioquinol, and occurs despite the presence of anti-inflammatory flumethasone in the preparation.

Our study has a number of limits. Our animal model, though similar to the human in many ways, forces us to extrapolate our results to humans. As the middle and inner ear anatomy and physiology in humans resembles that of the guinea pig, we feel that such an extrapolation is appropriate in this case. Second, ABR testing was done with a protocol initially designed for humans. Our equipment limits our evaluation to a maximum of $8,000 \mathrm{~Hz}$, though guinea pigs have been shown to respond to frequencies of up to $50,000 \mathrm{~Hz}$ [16]. Our functional evaluation of the basal turn of the cochlea is therefore sub-optimal. We partly compensate for this with SEM evaluation, which shows every turn equally. Furthermore, ototoxicity more commonly affects higher frequencies, thus when it is demonstrated in low frequencies, obtaining further proof is largely unnecessary. In addition, we are in front of a positive toxicity situation proving the unsafe use of Locacorten Vioforme independently of the frequencies affected.

Several topical are known to be safe and effective. This study, combined with a lack of proof of efficacy in the medical literature, shows that Locacorten Vioform should not be considered as an option in a patient with functional hearing in the presence of TM perforation or a pressure equalizing tube. However, because we identified a destructive effect of locacortene Vioforme on the inner ear for the tested frequencies, it is sufficient to exclude its application to treat mycotic external ear infection in the presence of TM perforation.
In conclusion, when applied directly to the middle ear of a guinea pig, Locacorten Vioform induces severe to profound hearing loss, similar to that observed with gentamycin. This occurs as a result of a rapid and severe pericochlear and cochlear inflammatory reaction with ossification. With a number of safer and more effective antimycotic agents available, Locacorten Vioform should not be used routinely in the treatment of otomycosis in the presence of tympanic tube or perforation.

\section{Conflicts of interest}

The authors have no financial conflicts of interest.

\section{REFERENCES}

1) Munguia R, Daniel SJ. Ototopical antifungals and otomycosis: a review. Int J Pediatr Otorhinolaryngol 2008;72:453-9.

2) Martin TJ, Kerschner JE, Flanary VA. Fungal causes of otitis externa and tympanostomy tube otorrhea. Int J Pediatr Otorhinolaryngol 2005;69:1503-8.

3) Jinn TH, Kim PD, Russell PT, Church CA, John EO, Jung TT. Determination of ototoxicity of common otic drops using isolated cochlear outer hair cells. Laryngoscope 2001;111:2105-8.

4) Tom LW. Ototoxicity of common topical antimycotic preparations. Laryngoscope 2000;110:509-16.

5) Baylancicek S, Serin GM, Ciprut A, Sari M, Akdaş F, Tutkun A. Ototoxic effect of topical ciclopirox as an antimycotic preparation. Otol Neurotol 2008;29:910-3.

6) Marsh RR, Tom LW. Ototoxicity of antimycotics. Otolaryngol Head Neck Surg 1989;100:134-6.

7) Woods O, Saliba I. Effect of nystatin on guinea pigs' inner ear. Neurotox Res 2011;20:301-6.

8) Bareggi SR, Cornelli U. Clioquinol: review of its mechanisms of action and clinical uses in neurodegenerative disorders. CNS Neurosci Ther 2012;18:41-6.

9) Soltermann W. [Locacorten-vioform in dermatological practice]. Praxis 1966;55:1194-7.

10) Kohn D, Wixson S, White W, Benson G. Anesthesia and analgesia in laboratory animals. 1st ed. San Diego, CA: Academic Press;1997. p. 172 .

11) Snedecor GW, Cochran WG. Statistical methods. 8th ed. Ames, IA: Iowa State University Press; 1989.

12) Mgbor N, Gugnani HC. Otomycosis in Nigeria: treatment with mercurochrome. Mycoses 2001;44:395-7.

13) Than KM, Naing KS, Min M. Otomycosis in Burma, and its treatment. Am J Trop Med Hyg 1980;29:620-3.

14) List RJ, Flook EP, Tay HL. Tympanoplasty graft preparation using ear drops containing polyethylene glycol, flumethasone and clioquinol. J Laryngol Otol 2011;125:853-5.

15) Prezant TR, Agapian JV, Bohlman MC, Bu X, Oztas S, Qiu WQ, et al. Mitochondrial ribosomal RNA mutation associated with both antibiotic-induced and non-syndromic deafness. Nat Genet 1993;4:28994.

16) Heffner R, Heffner H, Masterton B. Behavioral measurements of absolute and frequency-difference thresholds in guinea pig. J Acoust Soc Am 1971;49:1888-95. 